\title{
Humidified nasal continuous positive airway pressure in obstructive sleep apnoea
}

\author{
A.M. Neill*, H.S. Wai*, S.P.T. Bannan*, C.R. Beasley", M. Weatherall ${ }^{\#}$, A.J. Campbell*
}

Humidified nasal continuous positive airway pressure in obstructive sleep apnoea. A.M. Neill, H.S. Wai, S.P.T. Bannan, C.R. Beasley, M. Weatherall, A.J. Campbell. (C)ERS Journals Ltd 2003.

ABSTRACT: Heated humidification of nasal continuous positive airway pressure (nCPAP) reduces upper airway symptoms and improves initial use in obstructive sleep apnoea syndrome (OSAS). The present study aimed to assess the effect of heated humidification of nCPAP on upper airway symptoms and initial use in obstructive sleep apnoea.

This study was of a randomised, crossover design. Subjects with polysomnographically confirmed OSAS were randomised to 3 weeks nCPAP treatment with heated humidification (nCPAP-humid) or placebo humidification (nCPAP pl-humid). Objective and subjective nCPAP use, upper airway symptoms, and treatment satisfaction were compared.

Thirty seven of 42 patients completed the protocol. nCPAP-humid reduced the frequency of adverse upper airway symptoms. nCPAP use over 3 weeks was greater with nCPAP-humid compared with nCPAP pl-humid. No difference was found between the treatment arms in terms of subjective treatment satisfaction or alertness.

Heated humidification of nasal continuous positive airway pressure reduces upper airway symptoms and is associated with a small increase in initial use but not subjective sleepiness or treatment satisfaction. The results support the use of heated humidification as a strategy to reduce side-effects related to continuous positive airway pressure but not routine initial use.

Eur Respir J 2003; 22: 258-262.
*WellSleep Sleep Investigation Centre and

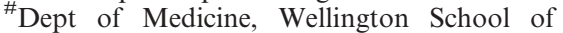
Medicine and Health Sciences, Wellington South, New Zealand.

Correspondence: A.M. Neill, Dept of Medicine, Wellington School of Medicine and Health Sciences, PO Box 7343, Wellington South, New Zealand.

Fax: 6449208861

E-mail: aneill@wnmeds.ac.nz

Keywords: Humidification

nasal continuous positive airway pressure obstructive sleep apnoea syndrome side-effects

Received: May 12002

Accepted after revision: April 112003

This study was funded by an Otago University Research Grant.
Obstructive sleep apnoea syndrome (OSAS) is a common condition [1, 2] characterised by loud snoring, repetitive obstructive breathing pauses during sleep and a range of daytime symptoms, including excessive daytime somnolence, reduced short-term memory and depression [3]. Nasal continuous positive airway pressure (nCPAP) alleviates the physiological abnormalities of OSAS [4] and is regarded as a firstline treatment. However, studies utilising objective monitoring devices demonstrate that long-term regular use may be as low as $46 \%$ with mean run times of $4.8 \mathrm{~h} \cdot$ night $^{-1}[5,6]$. Many factors are thought to influence continuous positive airway pressure (CPAP) use, including the intensity of patient support and follow-up [7], mask claustrophobia [5], previous palatal surgery $[8,9]$ and perceived lack of benefit [10]. nCPAP-related upper airway symptoms, including nasal congestion, dry nose or throat, sore throat and bleeding nose, affect between $30-50 \%$ of OSAS patients and limit long-term acceptance $[5,8,10,11]$.

Mouth leaks during nCPAP are common and offer a rational mechanism for the development of adverse upper airway symptoms as they cause unidirectional nasal airflow and progressive drying of the upper airway mucosa, release of inflammatory mediators, increased nasal mucosal blood flow and increased nasal resistance [12-14]. Heated humidification of inspired air attenuates the increase in mucosal blood flow and nasal resistance under experimental conditions [12, 13] and adverse upper airway symptoms in OSAS patients chronically treated with nCPAP $[9,15,16]$. In a recent crossover study, MASSIE et al. [15] demonstrated a small but significant improvement in CPAP use with heated humidified nCPAP (nCPAP-humid) compared to a 2-week washout period but no difference compared to CPAP with a cold passover humidifier [15]. As this study did not utilise a placebo intervention, the outcome variables in both humidification (heated and cold passover) arms were subject to a potentially significant placebo effect. PEPIN et al. [11] reported no difference in side-effects between patients who used humidity and those who did not, but no information was given regarding the type of humidity or selection criteria for humidity treatment, making it difficult to draw a firm conclusion. The addition of heated humidification to CPAP therapy requires systematic evaluation given the additional cost, set-up and cleaning time. This study aimed to assess the effect of heated humidification on initial nCPAP use, upper airway symptoms and daytime alertness using a randomised, crossover design.

\section{Methods}

\section{Subjects}

Adult patients were recruited from a sleep clinic population with newly diagnosed OSAS of sufficient severity to warrant treatment with nCPAP [17]. All patients were evaluated clinically to identify and treat surgically/medically correctable upper airway pathology including allergic rhinitis and polyposis. Patients were excluded if they had significant nasal 
obstruction (unable to breathe via a nasal route) or were unable to tolerate nCPAP during the titration study, required treatment for respiratory tract infection in the previous month, were mentally or legally incapacitated or suffered significant comorbidity, including psychiatric or cerebrovascular disease and respiratory or cardiac failure. Patients requiring supplemental oxygen were excluded. Study patients provided written consent and were informed that the study was designed to assess the effect of "two different levels of humidity" during nCPAP therapy. Patients were not told that CPAP use would be objectively monitored. At the completion of the study, each patient was debriefed with regard to compliance monitoring. Ethical approval was obtained from the Wellington Ethics Committee.

\section{Questionnaires}

At enrolment, patients completed questionnaires to document medical history, upper airway symptoms, atopy, medications, previous surgery to the nose or throat and sleepiness by Epworth Sleepiness Score (ESS) [18]. Specific chronic upper airway symptoms were recorded: dry nose; discharging or runny nose; congested or blocked nose; bleeding nose; reduced sense of smell; sinus infections; sinus pain or headaches; dry throat; sore throat; hoarse voice or cough.

At the end of each treatment arm patients repeated the questionnaire to assess specific upper airway symptoms and problems related to the use of the nCPAP device. A $10-\mathrm{cm}$ Likert scale was employed to measure satisfaction with the therapy, refreshment on waking, memory and ability to concentrate. Patients were asked to place a mark on the line to reflect the change in symptom. The midpoint represented no change, the far left worst ever and the far right best ever.

\section{Polysomnography and nasal continuous positive airway pressure titration}

Diagnostic polysomnography and nCPAP titration were undertaken at a tertiary level sleep laboratory (Wellington School of Medicine Sleep Investigation Centre, Bowen Hospital, New Zealand). All physiological signals were recorded on computer using an S-Series Sleep System (Compumedics P/L, Melbourne, Australia). Measurements included electroencephalogram (EEG) $\mathrm{C}_{4} \mathrm{~A}_{1}$ and $\mathrm{C}_{3} \mathrm{~A}_{2}$, electro-oculogram (EOG), and submental electromyogram (EMG) by surface electrodes, leg movements, sound (integrating sound level meter; Rion Co. Ltd, Tokyo, Japan), electrocardiogram (ECG), nasal pressure, ribcage and abdominal wall motion (piezoelectric sensors) and body position (mercury switch transducer). Oxyhaemaglobin saturation was measured by pulse oximetry (Satlite trans; Datex Engstrom, Helsinki, Finland).

nCPAP was manually titrated without added humidity by an experienced polysomnography technician. Titration protocols consisted of a $2-\mathrm{cm}$ increase in pressure for obstructive apnoea and a 1-cm increase for hypopnoea, snoring or desaturation. The final level of therapeutic CPAP was determined by a sleep physician. Patients underwent either a fullnight diagnostic CPAP followed by full-night nCPAP titration study or a split-night study (severe OSAS confirmed during first sleep cycle followed by nCPAP titration). Studies were manually scored for sleep stage [19] and arousals [20]. Definitions used for respiratory events included the following. Obstructive apnoea: cessation of airflow for $\geqslant 10 \mathrm{~s}$ accompanied by an ongoing respiratory effort. Central apnoea: cessation of airflow and respiratory effort for $\geqslant 10 \mathrm{~s}$. Mixed apnoea: combination of an obstructive and central apnoea lasting $\geqslant 10$ s. Hypopnoea: $>50 \%$ decrease in two of the three respiratory channel amplitudes (nasal pressure, chest or abdominal effort) for $\geqslant 10 \mathrm{~s}$. OSAS severity was expressed in terms of a respiratory disturbance index (RDI) (RDI= number apnoea+hypopnoea/hour of sleep).

\section{Study design}

A randomised crossover design was employed with the two 3-week treatment arms separated by a 3-day washout on no therapy. A treatment arm duration of 3 weeks was used as this has been shown to predict subsequent long-term CPAP use $[5,21]$. The patients were randomly assigned to nCPAP treatment (Sullivan Elite; ResMed Ltd, Sydney, Australia) with either heated humidity (CPAP-humid) or placebo humidity (nCPAP pl-humid). A nasal mask (Mirage or Bubble Cushion; ResMed Ltd) was fitted by an experienced sleep technician and used for the duration of the study. Standard equipment instructions and an educational video regarding the use of nCPAP were provided. nCPAP-humid was achieved by passing air from the CPAP machine through a heated water chamber (HC100; Fisher and Paykel HealthCare Ltd, Auckland, New Zealand). A setting of 2 was used as the authors previously found that higher settings resulted in significant precipitation into the mask or tubing. Patients were instructed not to change the setting unless water was collecting in the mask or tubing which would prompt reduction by 0.5 . The authors attempted to create a placebo humidification arm in which the $\mathrm{HC} 100$ unit was used without the heating unit turned on and the chamber was used without added water connected via tubing to the CPAP machine. During the 12- month study the mean monthly relative humidity readings at $06: 00 \mathrm{~h}$ were $85 \pm 3 \%$ (range $79-89 \%$ ) and the mean monthly temperature was $13 \pm 1^{\circ} \mathrm{C}$ (range $9.1-19.3^{\circ} \mathrm{C}$ ).

Patients were contacted by an experienced sleep technician after the first, seventh and fourteenth night of each treatment arm for support in accordance with the usual protocol for initiating CPAP. An investigator blinded to research treatment interviewed the patients at the end of each treatment arm (questionnaire, subjective and objective compliance (Scan Software; ResMed Ltd) and at the end of the washout period (questionnaire).

\section{Statistical analysis}

A power calculation based on a parallel design assumed a SD for the primary outcome variable (compliance) of $1.97 \mathrm{~h}$ [5]. A sample size per treatment arm of 42 has an $80 \%$ power to detect a difference of $1.22 \mathrm{~h}$ at a type-one error rate of $5 \%$. This represents $25 \%$ of the mean compliance of $4.88 \mathrm{~h}$ from another study [5]. As this trial had a crossover design this sample size estimate was conservative.

Data was entered into a computer database and statistical analysis was performed. A per protocol analysis was performed rather than an intention-to-treat analysis. For the primary outcome variable, "Proc Mixed" was used to fit a mixed linear model treating subjects as random effects and taking into account the repeated measurements of this variable $[22,23]$. Period, treatment, time of measurement and treatment by time of measurement effects were fitted in the first model and the treatment by time of measurement effect was omitted in the second model. Normality assumptions, based on analysis of residuals, were met.

The nasal symptoms were analysed in two ways. First, by treating the total count of symptoms as an outcome variable. 
Table 1.-Demographical and polysomnographical variables

\begin{tabular}{lc}
\hline Parameter & Mean \pm SD (range) \\
\hline M:F & $33: 4$ \\
Age yrs & $48.7 \pm 13.0(24-77)$ \\
BMI kg $\cdot \mathrm{m}^{-2}$ & $34.8 \pm 6.7(23-57)$ \\
Neck size cm & $43.3 \pm 3.2(38-51)$ \\
RDI events $\cdot \mathrm{h}^{-1}$ & $50.1 \pm 25.7(9-122)$ \\
Arousal index arousals $\cdot \mathrm{h}^{-1}$ & $39.1 \pm 22.0(6-121)$ \\
Average desaturation $\%$ & $8.41 \pm 3.58(3-17)$ \\
Minimum saturation $\%$ & $74.8 \pm 8.8(55-90)$ \\
CPAP pressure cm $\mathrm{CH}_{2} \mathrm{O}$ & $10.0 \pm 2.3(6-14)$ \\
ESS & $12.1 \pm 5.1(1-23)$ \\
\hline
\end{tabular}

n=37. M:F: male:female ratio; BMI: body mass index; RDI: respiratory disturbance index; CPAP: continuous positive airway pressure; ESS: Epworth Sleepiness Score.

This was analysed using a log-linear model that took into account the repeated measurements and the single baseline measurement using the SAS procedure "Proc Genmod". Secondly, each individual symptom was treated as a categorical variable and a method of conditional logistic regression was performed using the SAS procedure "Proc Logistic" for analysis of matched pairs [24]. The visual analogue scale scores were analysed using the "Proc Mixed" procedure after logistic transformation to meet normality assumptions.

\section{Results}

Of the forty-two patients initially enrolled five were unable to complete the study protocol. Three developed acute respiratory tract infections (two while receiving nCPAP plhumid and one while receiving nCPAP-humid). One patient breached the protocol by continuing to use water in the humidification chamber during the nCPAP pl-humid arm of the trial and another patient was lost to follow-up. Thirtyseven patients (four females and 33 males) aged 47.3 $\pm 13 \mathrm{yrs}$ with moderate-to-severe obstructive sleep apnoea (RDI: $50.1 \pm 25.7 \mathrm{~h}^{-1}$ ) completed the study protocol. Seventeen patients were randomised to nCPAP pl-humid first and 20 patients to nCPAP-humid first. Demographical and polysomnographical results are detailed in table 1.

\section{Subjective effect of treatment and patient preference}

nCPAP in both arms of the study reduced sleepiness measured by ESS from baseline $12.1 \pm 5.1$ to $4.1 \pm 2.1$ with nCPAP-humid $(\mathrm{p}<0.01)$ and to $4.5 \pm 3.1$ with CPAP with placebo humidity (nCPAP pl-humid) $(\mathrm{p}<0.01)$. There was no significant difference between the two arms $(p=0.37)$. The effect of CPAP treatment on memory, ability to concentrate, feeling refreshed on waking and overall treatment satisfaction were not different between the two arms of the study (table 2). nCPAP with heated humidification was preferred by $51 \%$ of patients; no humidification was preferred by $35 \%$ of patients; $14 \%$ were undecided $(\mathrm{p}=0.24)$.

\section{Effect of treatment on upper airway symptoms}

Upper airway symptoms were common, with $34(92 \%)$ patients reporting at least one symptom (table 3 ). The frequency of upper airway symptoms was reduced by nCPAPhumid compared to nCPAP pl-humid by 1.4 symptoms $(95 \%$ confidence interval (CI) 1.07-1.83, $\mathrm{p}<0.02$ ). The point differences in eight of 11 upper airway symptoms (table 3 ) favoured a positive treatment benefit for humidification but this did not reach statistical significance.

Table 2.-Outcome variables after 3 weeks nasal continuous positive airway pressure (CPAP) with and without heated humidification

\begin{tabular}{lrrr}
\hline & nCPAP-humid & nCPAP pl-humid & p-value \\
\hline Likert scale $^{\#}$ & & & \\
Memory & $73.2 \pm 14.1$ & $71.6 \pm 17.1$ & 0.88 \\
Ability to concentrate & $76.7 \pm 14.0$ & $75.2 \pm 15.9$ & 0.41 \\
Refreshed on awakening & $78.15 \pm 14.1$ & $78.9 \pm 16.9$ & 0.61 \\
Treatment satisfaction \% & $80.8 \pm 14.5$ & $78.2 \pm 16.4$ & 0.73 \\
\hline
\end{tabular}

Data are presented as mean \pm SD. nCPAP-humid: heated humidity continuous positive airway pressure; nCPAP humid-pl: placebo humidity continuous positive airway pressure. ${ }^{\#}$ : $0 \%$ (worst ever) to $100 \%$ (best ever); ${ }^{\uparrow}: 0 \%$ (very dissatisfied) to $100 \%$ (very satisfied).

Table 3. - Upper airway symptoms pre- and post-continuous positive airway pressure heated-humid (nCPAP-humid) and CPAP placebo-humid (nCPAP pl-humid)

\begin{tabular}{|c|c|c|c|c|c|}
\hline Symptom & Baseline & nCPAP-humid & nCPAP pl-humid & OR $(95 \% \mathrm{CI})^{\#}$ & $\mathrm{p}$-value \\
\hline Dry nose & 16 & 24 & 41 & $3(0.81-11.1)$ & 0.10 \\
\hline Discharging or runny nose & 19 & 22 & 27 & $2(0.50-8.0)$ & 0.33 \\
\hline Congested or blocked nose & 51 & 51 & 67 & $7(0.90-56.9)$ & 0.07 \\
\hline Bleeding nose & 5 & 11 & 11 & $1(0.14-701)$ & 1 \\
\hline Reduced sense of smell & 27 & 16 & 22 & $1.7(0.40-7.0)$ & 0.48 \\
\hline Sinus infection & 27 & 14 & 14 & $0.7(0.10-4.0)$ & 0.67 \\
\hline Sinus pain or headache & 41 & 14 & 27 & $2.7(0.71-10.1)$ & 0.15 \\
\hline Dry throat & 68 & 27 & 46 & $2.8(0.88-8.6)$ & 0.08 \\
\hline Sore throat & 24 & 5 & 16 & $5(0.58-42.8)$ & 0.14 \\
\hline Hoarse voice & 21 & 10 & 14 & $1.7(0.40-7.0)$ & 0.48 \\
\hline Cough & 41 & 31 & 24 & $0.6(0.14-2.5)$ & 0.48 \\
\hline Median symptom frequency & 3 & 2 & 3 & $1.4(1.07-1.83)$ & $<0.02$ \\
\hline
\end{tabular}

Data are presented as per cent of subjects. OR: odds ratio; CI: confidence interval. ${ }^{\#}$ : OR for response to a question being negative on nCPAP-humid arm versus nCPAP pl-humid. 
Table 4. - Objective nasal continuous positive airway pressure (nCPAP) use with and without heated humidification by week

nCPAP-humid nCPAP pl-humid

\begin{tabular}{|c|c|c|}
\hline Total use $\mathrm{h} \cdot$ night $^{-1}$ (range) & $5.7 \pm 1.5(2.6-8.3)$ & $5.3 \pm 1.7(1.4-8.1)$ \\
\hline Week $1 \mathrm{~h} \cdot$ night $^{-1}$ & $5.9 \pm 1.6$ & $5.4 \pm 1.6$ \\
\hline Week $2 \mathrm{~h} \cdot$ night $^{-1}$ & $5.8 \pm 1.5$ & $5.3 \pm 2.0$ \\
\hline Week $3 \mathrm{~h} \cdot$ night $^{-1}$ & $5.5 \pm 1.8$ & $5.3 \pm 1.9$ \\
\hline
\end{tabular}

Data are presented as mean \pm SD. nCPAP-humid: heated humidity nCPAP; nCPAP pl-humid: placebo humidity nCPAP.

\section{Nasal continuous positive airway pressure use}

Objectively measured nCPAP use was greater with nCPAPhumid $\left(5.7 \pm 1.5 \mathrm{~h} \cdot 24 \mathrm{~h}^{-1}\right)$ compared to nCPAP pl-humid $(5.3 \pm$ $\left.1.7 \mathrm{~h} \cdot 24 \mathrm{~h}^{-1}\right)\left(95 \%\right.$ CI for difference was $0.05-0.76 \mathrm{~h} \cdot$ night $^{-1}$, $\mathrm{p}=0.03$ ) when averaged over the 3 weeks. The treatment time by measurement time interaction did not reach statistical significance for objectively measured compliance at weekly time intervals (table 4). Patients subjectively overestimated their nCPAP pl-humid use by $1.48 \pm 1.65 \mathrm{~h} \cdot$ night $^{-1} \quad(\mathrm{p}<0.01)$ and nCPAP-humid use by $1.12 \pm 1.39 \mathrm{~h} \cdot$ night $^{-1} \quad(\mathrm{p}<0.01)$. Subjective CPAP use was not statistically different between therapies $(\mathrm{p}=0.57)$.

The presence of specific upper airway symptoms and the frequency of upper airway symptoms at baseline were not related to objectively measured compliance $(\mathrm{p}=0.23)$ or final treatment preference $(\mathrm{p}=0.62)$.

\section{Discussion}

This study demonstrates that the addition of heated humidification reduces the frequency of adverse upper airway symptoms and modestly improves initial nCPAP use over 3 weeks. However, these effects were not associated with a greater improvement in daytime sleepiness or treatment satisfaction. There was no clear preference for either therapy, with a similar proportion of patients preferring humidity $(51 \%)$ or no humidity $(35 \%)$ and with some undecided $(14 \%)$.

It was found that the addition of humidification increased nCPAP use during a 3 -week period by an average of $24 \mathrm{~min} \cdot$ night $^{-1}$. Previous studies of objectively measured nCPAP use have shown that compliance with the device over the first 2-4 weeks predicts subsequent longer term use $[5,21]$. However, given the short duration of this study and the magnitude of the difference, caution is advised in interpreting the clinical significance of this difference in CPAP use. Under the conditions of a "Proc Mixed" linear model for repeated measurements, separate weekly CPAP compliance did not reach statistical significance (table 4). However, the magnitude of the difference appeared to be greatest during the first week of treatment, raising the possibility that the compliance difference may be short term.

There are clearly many important factors involved in determining long-term nCPAP acceptance. It was hypothesised that the addition of heated humidification would improve objectively measured initial nCPAP compliance and reduce adverse upper airway symptoms when compared to CPAP without humidification. The rationale for using humidification has been well developed. During normal nasal breathing conditions the nasal mucosa recovers approximately onethird of water delivered to the inspired gases from expired gases. nCPAP significantly reduces the relative humidity of inspired air, particularly in the presence of mouth leaks in normal, awake volunteers [12] and awake and asleep OSAS patients [25]. Unidirectional airflow promoted by mouth leaks overwhelms the capacity of the nasal mucosa to heat and humidify inspired air, leading to progressive nasal mucosal drying, release of inflammatory mediators, increased nasal mucosal blood flow and increased nasal resistance [12, 13]. Humidification of inspired gases has been shown to attenuate these effects in normal awake volunteers $[12,13]$ and in OSAS patients using long-term nCPAP [25].

MORTIMORE et al. [26] have shown that the use of a full face mask reduces the complaint of dry nose and mouth but most patients felt that the nasal mask was more comfortable [26]. In addition, SANDERS et al. [27] report that only two of 17 patients would accept the full face mask long term.

In this study the frequency of upper airway symptoms decreased when CPAP was used with heated humidification compared to nCPAP pl-humid conditions. The effect would appear to be clinically relevant but could not be directly related to improved use. The point differences in eight of 11 upper airway symptoms (table 3 ) favoured a positive treatment benefit for humidification but this did not reach statistical significance. This is most likely to be due to the study being underpowered for individual upper airway symptoms (which were not a primary outcome variable). Improved usage or treatment preference could not be predicted either from the frequency of initial or specific upper airway symptoms. Either other factors associated with humidification were important or patients to some extent "put up with" some adverse symptoms which were not of sufficient severity to influence CPAP use. It should be noted that adverse upper airway symptoms persisted at a similar frequency to baseline when nCPAP was used without added humidity, arguing against short-term adaptation. It is possible that the persistence of these symptoms could eventually lead to treatment failure. A longer term randomised placebo-controlled study would help address this issue.

The increase in nCPAP use with humidification observed in this study did not translate into improved levels of alertness on waking or a greater reduction in sleepiness scores. There are a number of potential reasons for this, including the relatively small effect size with a $24 \mathrm{~min} \cdot$ night $^{-1}$ average increase in CPAP use and possibly the relative insensitivity of the tools used in this study to assess symptoms. The authors did not objectively measure sleepiness by multiple sleep latency test or maintenance of wakefulness test. MASSIE et al. [15] reported similar mean improvements in nCPAP use when comparing heated humidity to a washout period without humidity with improvements in "waking feeling refreshed" but did not control for a placebo effect. The effects of cold passover humidification in this study were very similar to heated humidification for CPAP compliance, treatment satisfaction and ESS. This could be due to a placebo effect or due to cold passover humidification. Whilst not specified in their methodology, patients using this adjuvant to CPAP frequently use warm water.

In an attempt to control for a placebo effect, all study patients were naïve to nCPAP and the use of adjuvant heated humidification. During each treatment arm they were given simple instructions regarding the use of the equipment, the same follow-up protocol and told that the purpose of the study was to compare the effect of CPAP treatment at "two levels of humidity". The investigator administering the questionnaires was blinded to treatment arm. The subjective assessment of CPAP use by treatment diary overestimated actual use under both conditions but did not differ between arms, supporting the view that patients believed they were in fact getting a similar amount of treatment.

The results of this study support the hypothesis that the addition of heated humidification reduces upper airway symptoms but the magnitude of the difference in initial use was modest and not associated with improved levels of 
sleepiness or treatment satisfaction. The results do not support the routine initial use of heated humidification with nasal continuous positive airway pressure. A more logical approach would be to use heated humidification as one of several strategies, including passover humidification, chin strap or full face-mask use, in those subjects who complain of adverse upper airway symptoms (particularly dry throat and nose) or who are poorly compliant and might otherwise stop treatment.

Acknowledgements. The authors would like to thank G. Richards for assistance developing the upper airway symptom questionnaire.

\section{References}

1. Lavie P. Incidence of sleep apnea in a presumably healthy working population: a significant relationship with excessive daytime sleepiness. Sleep 1983; 4: 312-318.

2. Young T, Palta M, Dempsey J, Skatrud J, Weber S, Badr S. The occurrence of sleep-disordered breathing among middleaged adults. NEJM 1993; 328: 1230-1235.

3. Cheshire K, Engleman H, Deary I, Shapiro C, Douglas N. Factors impairing daytime performance in patients with sleep apnea/hypopnea syndrome. Arch Int Med 1992; 152: 538-541.

4. Sullivan C, Berthon-Jones M, Issa F, Eves L. Reversal of obstructive sleep apnoea by continuous positive airway pressure applied through the nares. Lancet 1981; 1: 862-865.

5. Kribbs N, Pack A, Kline L, et al. Objective measurement of patterns of nasal CPAP use by patients with obstructive sleep apnea. Am Rev Respir Dis 1993; 147: 887-895.

6. Engleman H, Martin S, Douglas N. Compliance with CPAP therapy in patients with the sleep apnoea/hypopnoea syndrome. Thorax 1994; 49: 263-266.

7. Hoy C, Vennelle M, Kingshott R, Engleman H, Douglas N. Can intensive support improve continuous positive airway pressure use in patients with the sleep apnea/hypopnea syndrome? Am J Respir Crit Care Med 1999; 159: 1096-1100.

8. Waldhorn R, Herrick T, Nguyen M, O'Donnell A, Sodero J, Potolicchio S. Long term compliance with nasal continuous positive airway pressure therapy of obstructive sleep apnea. Chest 1990; 97: 33-38.

9. Rakotonanahary D, Pelletier-Fleury N, Gagnadoux F, Fleury B. Predictive factors for the need for additional humidification during nasal continuous positive airway pressure therapy. Chest 2001; 119: 460-465.

10. Hoffstein V, Viner S, Mateika S, Conway J. Treatment of obstructive sleep apnea with nasal continuous positive airway pressure. Patient compliance, perception of benefits, and side effects. Am Rev Respir Dis 1992; 145: 841-845.

11. Pepin J, Leger P, Veale D, Langevin B, Robert D, Levy P.
Side effects of nasal continuous positive airway pressure in sleep apnea syndrome. Chest 1995; 107: 375-381.

12. Richards $\mathrm{G}$, Cistulli $\mathrm{P}$, Ungar R, Berthon-Jones $\mathrm{M}$, Sullivan C. Mouth leak with nasal continuous positive airway pressure increases nasal airway resistance. $\mathrm{Am}$ J Respir Crit Care Med 1996; 154: 182-186.

13. Hayes M, McGregor F, Roberts D, Schroter R, Pride N Continuous nasal positive airway pressure with a mouth leak: effect on nasal mucosal blood flux and geometry. Thorax 1995; 50: 1179-1182.

14. Ohki M, Usui N, Kanazawa H, et al. Relationship between oral breathing and nasal obstruction in patients with obstructive sleep apnea. Acta Otolaryngol 1996; 523: 228 230

15. Massie C, Hart R, Peralez K, Richards G. Effects of humidification on nasal symptoms and compliance in sleep apnea patients using continuous positive airway pressure. Chest 1999; 116: 403-408.

16. Wiest G, Lehnert G, Bruck W, Meyer M, Hahn E, Ficker J. A heated humidifier reduces upper airway dryness during continuous positive airways pressure therapy. Respir Med 1999; 93: 21-26.

17. Australian Health Technology Advisory Committee. The effectiveness and cost-effectiveness of nasal continuous positive airway pressure (nCPAP) in the treatment of obstructive sleep apnea in adults. Canberra, The National Health and Medical Research Council and the New Zealand Ministry of Health, 1997.

18. Johns MW. A new method for measuring daytime sleepiness: The Epworth Sleepiness Scale. Sleep 1991; 14: 540-545.

19. Rechtschaffen A, Kales AE. A Manual of Standardized Terminology, Techniques and Scoring System for Sleep Stages of Human Subjects. University of California, Brain Information Service/Brain Research Institute, 1968.

20. American Sleep Disorders Association. EEG Arousals: scoring rules and examples. Sleep 1992; 15: 173-184.

21. Reeves-Hoche M, Meck R, Zwillich C. Nasal CPAP: An objective evaluation of patient compliance. Am J Respir Crit Care Med 1994; 149: 149-154.

22. Brown H, Prescott R. Applied mixed models in medicine. Chichester, J Wiley and Sons, 1999.

23. Senn S. Cross-over trials in clinical research. Chichester, J Wiley and Sons, 2002.

24. Agresti A. An introduction to categorical data analysis. New York, J Wiley and Sons, 1996.

25. Martins de Araujo M, Vieira S, Vasquez E, Fleury B. Heated humidification or face mask to prevent upper airway dryness during continuous positive airway pressure therapy. Chest 2000; 117: 142-147.

26. Mortimore I, Whittle A, Douglas N. Comparison of nose and face mask CPAP therapy for sleep apnoea. Thorax 1998 ; 53: 290-292.

27. Sanders M, Kern N, Stiller R, et al. CPAP therapy via oronasal mask for obstructive sleep apnea. Chest 1994; 106: 774-779. 